\title{
Good Computational Practice in the Assignment of Absolute Configurations by TDDFT Calculations of ECD Spectra
}

\author{
Gennaro Pescitelli*[a] and Torsten Bruhn*[b]
}

\begin{abstract}
Quantum-mechanical calculations of chiroptical properties have rapidly become the most popular method for assigning absolute configurations (AC) of organic compounds, including natural products. Black-box TDDFT calculations of ECD spectra are nowadays easily accessible to non-experts.
\end{abstract}

However, an uncritical attitude may easily deliver a wrong answer. We present to the Chirality Forum a discussion on what can be called a good computational practice in running TDDFT ECD calculations, highlighting the most crucial points with several examples from the recent literature.

Keywords: electronic circular dichroism, natural products, density functional theory, chiroptical spectroscopy, structure elucidation

\section{Introduction}

The rapid development of computational power ${ }^{1}$ has, among many other things, revolutionized the way chemists and other scientists face with the fundamental problem of assigning the absolute configuration (AC) of new molecules. ${ }^{2}$ Once based exclusively on the Bijvoet method or chemical correlations, this problem has lately become the main purpose of chiroptical spectroscopies, first of all electronic circular dichroism (ECD). ${ }^{3}$ However, for a long time the interpretation of ECD spectra of new compounds has been based entirely on empirical or semiempirical methods such as spectral comparisons or sector rules. $^{4,5}$ Not surprisingly then, non-empirical methods of analysis such as the exciton chirality have become quickly popular and spurred a lot of research., ${ }^{6,7}$ The revolution, or renaissance of chiroptical spectroscopies, ${ }^{8}$ however arrived when the ab initio theoretical simulation of chiroptical properties including ECD, optical rotation (OR), vibrational CD (VCD), Raman optical activity (ROA), and so on, became a concrete possibility for real-life molecules such as natural products and transition metal complexes. ${ }^{9-13}$ Quantum-mechanical (QM) calculations of chiroptical spectra make it possible to assign AC's without the need for any reference system or any chemical derivatization, and often without the necessity of establishing the molecular physico-chemical mechanisms responsible for the observed property (the so-called mechanisms of optical activity). In the specific context of ECD, where a prediction of excited states is necessary for the simulation, a step forward has been taken with the advent of the very cost-effective time-dependent density functional theory (TDDFT) method. $^{14}$ The efficiency of TDDFT allows the simulation of the ECD spectrum of a medium-size molecule on a desktop $P C$ in a reasonable time, and the availability of TDDFT ECD calculations in commercial and freely available software has opened the way to a wide usage also by nonexperts. ${ }^{15}$ The other side of the coin is that a black-box usage of computational tools may easily have unexpected negative consequences on the results. ${ }^{16}$ This is especially true when the determination of the AC is concerned: it is obvious that "there are only two possible conclusions in absolute configurational studies: the conclusion is either correct or wrong". ${ }^{6}$ The aim of the present contribution to the Chirality Forum is to share our thoughts on what can be called a good computational practice in assigning the AC of organic compounds by means of TDDFT ECD calculations. We will first present a typical flowchart to achieve the AC assignment task, then we will discuss each step with a few examples aimed at highlighting the most critical aspects of the outlined procedure.

\section{Results and Discussion}

\section{A TYPICAL COMPUTATIONAL FLOWCHART}

The computational study ultimately leading to an ECD calculation of a given molecule starts with the generation of input structures. While the molecular conformation is determined on the way during the computational procedure, all other structural features must be known in advance, including molecular constitution (the so-called "planar" structure) and, very importantly, the relative configuration $(\mathrm{RC})$ of the various chirality elements in the molecule. In principle, this latter piece of information may also be determined by chiroptical spectroscopies, and especially by a combination thereof. ${ }^{17,18}$ However, we highly recommend the use of independent tools, including X-ray crystallography (whenever available) and NMR spectroscopy to elucidate as much as possible of the RC. In most cases a careful evaluation of a full set of NMR data sensitive to three-dimensional structure such as scalar $\mathrm{J}$ couplings (especially ${ }^{3} \mathrm{~J}_{\mathrm{HH}}$ and ${ }^{3} \mathrm{~J}_{\mathrm{CH}}$ ) and NOE effects will furnish the relative stereochemistry. ${ }^{19}$ It must be stressed that when flexible molecules are concerned, "real" molecular models and not just planar structures should be used to rationalize and

[a] Prof. G. Pescitelli

Dipartimento di Chimica e Chimica Industriale

Università di Pisa

via Moruzzi 13, I-56124 Pisa, Italy

E-mail: gennaro.pescitelli@unipi.it

[b] Dr. Torsten Bruhn

Institut für Organische Chemie

Universität Würzburg Am Hubland

97074 Würzburg, Germany

E-mail: torsten.bruhn@uni-wuerzburg.de

Received: ((will be filled in by the editorial staff))

Revised: ((will be filled in by the editorial staff)) Published online: ((will be filled in by the editorial staff)) 
interpret NOE data. So, we assume that the AC of a molecule with known constitution and RC must be determined, and that a starting molecular model of such a molecule (with the correct $\mathrm{RC}$ !) has been generated by a molecular modeling software with an initially arbitrary AC. The typical steps of the ECD computational study are the following (see flowchart in Figure 1). ${ }^{15,20}$

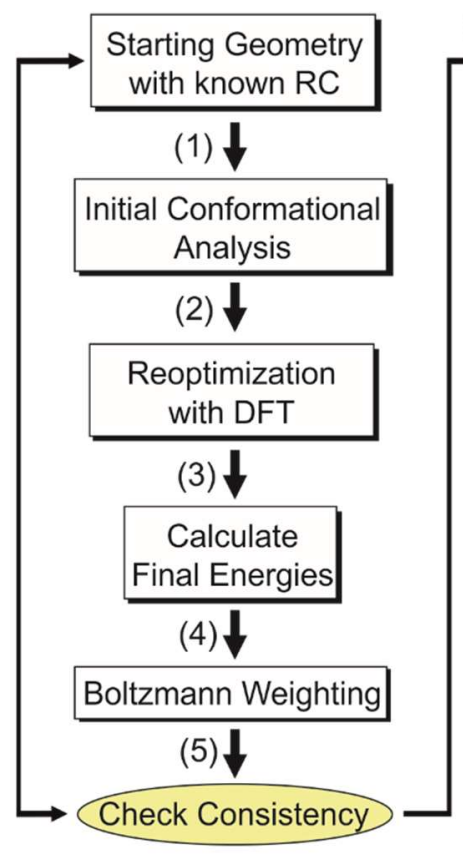

(6)

Calculate UVIECD

(7)

Convert Sticks to Curves by Gaussians

(8)

\begin{tabular}{|c|}
\hline $\begin{array}{c}\text { Prepare Overall } \\
\text { Spectra }\end{array}$ \\
\hline
\end{tabular}

(9)

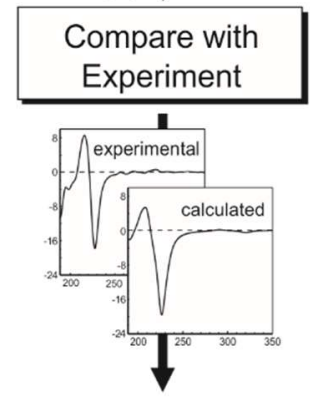

Absolute Configuration

FIGURE 1 Flowchart for the typical steps in ECD calculations.

(1) Perform a thorough conformational search at a low computational level, typically molecular mechanics (MM) with a good force field (FF) such as MMFF (Merck Molecular FF), ${ }^{21}$ to investigate the conformational ensemble. This may be done with a conformational analysis protocol such as Monte Carlo or molecular dynamics. ${ }^{22}$ It is important that all rotatable bonds are varied and all endocyclic atoms are puckered during the conformational search, in order not to miss any possible conformer. ${ }^{16}$

(2a) Run a preliminary geometry optimization of all conformers found in step (1) at an intermediate level of calculation. This is typically done using the B3LYP/6-31G(d) combination, however the use of a more efficient functional for structure and energetics is recommended, ${ }^{23}$ possibly including dispersion correction, ${ }^{24}$ in combination with a double- $\zeta$ basis set. A different time-efficient approach - especially for large molecules - giving geometries of high quality is the PBEh-3c method proposed by Grimme et al. ${ }^{25}$ In this step the MM-structures are screened and a restricted number of low-energy conformations is obtained, falling in an energy window within a certain threshold, i.e. 5 or $10 \mathrm{kcal} / \mathrm{mol}$.

(2b) Run a more refined geometry optimization of the structures selected in (2a) at a higher level of calculation. This may include: (a) a larger basis set such as a triple- $\zeta$ or with additional polarization or even diffuse functions. In general, Ahlrichs' basis sets such as def2-SVP or def2-TZVP26 are better suited than Pople's family (e.g. 6-31G(d) and following); (b) a solvent model such as the Polarizable Continuum Model (PCM), ${ }^{27} \mathrm{COSMO},{ }^{28}$ or SMD. ${ }^{29}$
(3) Optionally, the set of low-energy structures found in step (2) can be submitted to single-point energy calculations at even higher level (for example using a larger basis sets than in point (2b), or including a solvent model), and/or to frequency calculations to verify their nature of true energy minima and to obtain free energies or zero-point corrected (ZPC) energies. Frequency calculations must be run at the same level of geometry optimizations.

(4) Apply Boltzmann distribution to the set of low-energy minima obtained in steps (2) or (3), by using internal, ZPC or free energies. ${ }^{20,30,31} \mathrm{~A}$ final set of structures is thus obtained together with their respective Boltzmann populations at $298 \mathrm{~K}$ or $300 \mathrm{~K}$. Those falling above a certain population threshold, say $1 \%$, need to be considered in the following steps.

(5) Check the consistency between the calculated conformational ensemble and the whole set of conformationdependent NMR data ( $J$-couplings, NOE contacts, ring-current shifts). This - in our opinion - very important step is most often overlooked in the current literature. This computational/ experimental consistency check may be hampered or greatly complicated for very flexible molecules, for which experimental data are the average of several low-energy conformations. Such a situation may greatly benefit from low-temperature NMR experiments, which in favorable cases also allow a direct quantification of conformers. ${ }^{32}$ Even in the most difficult cases however one should always verify that the most populated conformers do not contradict experimental results, and viceversa. If this happens, the conformational analysis should be checked for errors and repeated; on the other hand, it may also be a hint of wrong structural assignment, for example of the RC.

(6) Calculate the absorption and ECD spectra for all populated conformers found in step (4). This is often the most computationally demanding step in the whole procedure, and for which the choice of the level of calculation is most crucial. ${ }^{9,13,14,20,33,34}$ Restricting only to the TDDFT method, both the functional and the basis set should be chosen carefully. Most importantly, one should never use just a single functional/basis set combination, and different combinations should always be explored. As for the functional, at least two families of functionals, i.e. hybrid and range-separated functionals, should both be tested. Popular hybrid functionals with increasing amounts of "exact" or HF exchange are for example B3LYP (20\% HF), PBE0 (25\%), M06 (27\%), BH\&HLYP (50\%), and M06-2X (54\%). ${ }^{35}$ Range-separated functionals such as CAM-B3LYP and $\omega B 97 X$ perform often better than hybrid ones in ECD calculations and should always be considered. Both the HF percentage and the range separation affect, among other things, the absolute and relative transition energies, ${ }^{36}$ therefore a correct reproduction of the sequence of bands and their energies is one of the factors dictating the final choice (see "Choice of the TDDFT Functional" section below). As for the basis set, we recommend Ahlrichs' def2-SVP or def2-TZVP, ${ }^{26}$ or, more in general, basis sets of double or triple- $\zeta$ quality with a sufficiently wide set of polarization functions. Diffuse functions may be necessary whenever Rydberg states contribute substantially to the ECD spectrum. ${ }^{37}$ The basis set convergence may be easily verified by using one of the two methods discussed in paragraph "Choice of the TDDFT Basis Set" in the next section. In general, the weaker the computed (and experimental) ECD spectrum, the stronger will be its dependence on the calculation method, including functional, basis set, input geometry, and solvation model. An absorption/ECD calculation is essentially an excited-state 
calculation: the number of excited states (roots) to be included must be chosen in a way that the final calculated absorption and ECD spectra, after the wavelength correction (vide infra), extend well below the lowest observed wavelength.

(7) As the ECD calculation run in step (6) actually provides a list of rotational strengths as a function of wavelength or energy (stick plot), generate a true ECD spectrum by applying a bandshape to each rotational strength and sum over the whole spectral range. A Gaussian band-shape is normally used for electronic $\mathrm{CD}^{38}$ The choice of the bandwidth, namely the standard deviation of the Gaussian curve, is often done empirically on a best-fit basis; reasonable values for the socalled FWHM (full width at half maximum) are in the range 0.1$0.3 \mathrm{eV}$. The same applies to the absorption spectrum generated by computed dipolar or oscillator strengths.

(8) Calculate the final Boltzmann-averaged spectrum by weighting each component spectrum obtained in step (7) with the appropriate conformer Boltzmann weight estimated in step (4), and then by adding all weighted spectra to each other.

(9) Compare the experimental absorption/ECD spectrum with the final calculated spectrum obtained in step (8) over the whole available spectral range. In doing so, a wavelength shift may be applied to take into account a systematic over/underestimation of transition energies (sometimes this is called UV correction, as it is simpler to determine the systematic error from a comparison of experimental and calculated UV absorption spectra). If a good match is obtained, the assumed $A C$ is the correct one; if there is a good mirror-image relationship, the opposite AC is correct. Since the ECD spectra of two enantiomers are mirror images by definition, it is not necessary to run the calculation on both enantiomers; when this is done, however, an exact mirror-image relationship must result. Although the comparison between calculation and experiment is often done visually, we recommend the use of a tool allowing for a quantitative comparison. ${ }^{39,40}$ This will furnishing a similarity factor which, hopefully, should be sizably larger for the correct AC than the incorrect one (ideally, 1 vs. 0). If the comparison is poor for both possible enantiomers over the available spectral range, the computational procedure should be reexamined, starting first from the choice of functional/basis set combination in step (6) and eventually from the very first step (1) of conformer generation.

\section{EXAMPLES AND IN-DEPTH DISCUSSION}

\section{Geometry optimizations and relative energies}

One of the major problems connected with QM calculations of molecular properties is the widespread and often uncritical use of the B3LYP hybrid functional. ${ }^{41}$ In particular, geometry optimizations and related quantities such as relative internal energies are very commonly estimated at B3LYP/6-31G(d) level. This naïve approach has several drawbacks which cannot be fully treated here. Simply stated, several benchmark studies have demonstrated that B3LYP is not necessarily the most accurate functional for predicting structures and energies of standard organic molecules. ${ }^{23}$ Other largely available hybrid and range-separated functionals, including Truhlar M0x and HeadGordon wB97 family, perform better in general terms. ${ }^{42,43}$ Moreover, when non-covalent interactions come into play, a reliable prediction of conformational energies requires the inclusion of dispersion, either as a correction of normal hybrid or double-hybrid functionals. ${ }^{24,44,45}$ As an example, 2-(3-fluoro-( $p$ tolyl)propyl)naphthalene (1, Scheme 1) may exist in unfolded or folded conformations depending on the presence of an intramolecular $\pi$-stacking. Folded conformations are however totally overlooked by B3LYP calculations, while they turn out to be the most stable ones when B2GPPLYP-D3 is used for geometry optimizations in vacuo (Figure 2). As the two conformational families (unfolded and folded) have very different calculated ECD spectra, their relative population has a large impact on the average calculated ECD spectrum. ${ }^{10}$<smiles>COc1cc(O)c2c(c1)C(=O)C1=C(C2=O)[C@H](O)[C@](C)(O)[C@H](C(C)=O)[C@@H]1O</smiles>

SCHEME 1 Structures of (S)-2-(3-fluoro-( $p$-tolyl)propyl)naphthalene (1) and altersolanol L (2) mentioned in the text.

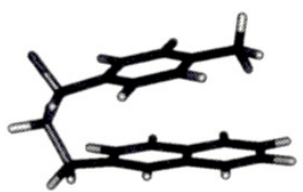

B2GPPLYP-D3: lowest-energy, $36 \%$ population B3LYP: $+4.43 \mathrm{kca} / \mathrm{mol}, 0 \%$ population

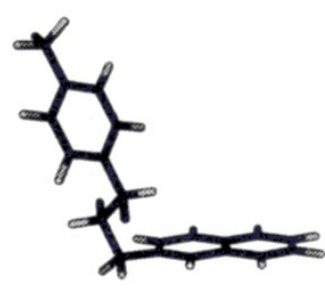

B2GPPLYP-D3:

$+1.47 \mathrm{kca} / \mathrm{mol}, 3 \%$ population

B3LYP:

lowest-energy, $89 \%$ population
FIGURE 2 The two lowest energy conformations calculated for (S)-2(3-fluoro-( $p$-tolyl)propyl)naphthalene (1, Scheme 1) at B2GPPLYPD3/def2-TZVPP and B3LYP/TZVPP' levels (left, folded, and right, unfolded) in vacuo. Adapted from ref. ${ }^{10}$, with permission.

TABLE 1 Boltzmann populations (in \%) at $300 \mathrm{~K}$ for the conformers of altersolanol $L(2)$ estimated at various levels of calculation (from geometry optimizations) ${ }^{46}$

\begin{tabular}{|c|c|c|c|c|c|}
\hline$\#^{a}$ & $\mathrm{MM}$ & $\begin{array}{l}\text { small } \\
\text { basis } \\
\Delta \mathrm{H}^{\mathrm{c}}\end{array}$ & $\begin{array}{l}\text { large } \\
\text { basis }^{b} \\
\Delta \mathrm{H}^{\mathrm{c}}\end{array}$ & $\begin{array}{l}\text { large } \\
\text { basis }^{\mathrm{b}} \\
\Delta \mathrm{H}^{\mathrm{c}}+\mathrm{PCM}^{\mathrm{d}}\end{array}$ & $\begin{array}{l}\text { large } \\
\text { basis }^{b} \\
\Delta \mathrm{G}^{\mathrm{c}}+\mathrm{PCM}^{\mathrm{d}}\end{array}$ \\
\hline 01 & 78.8 & 90.5 & 46.8 & 3.2 & 0.3 \\
\hline 02 & 15.9 & 0.0 & 0.2 & 0.6 & 0.4 \\
\hline 03 & 2.9 & 2.3 & 5.7 & 2.2 & 1.3 \\
\hline 04 & 1.6 & 6.2 & 42.6 & 62.6 & 71.3 \\
\hline 05 & 0.6 & 0.0 & 0.0 & 0.0 & 0.0 \\
\hline 06 & 0.1 & 0.0 & 0.2 & 0.0 & 0.0 \\
\hline 07 & 0.1 & 0.3 & 3.5 & 28.9 & 25.6 \\
\hline 08 & 0.1 & 0.6 & 0.5 & 0.5 & 0.4 \\
\hline 09 & 0.0 & 0.0 & 0.0 & 0.0 & 0.0 \\
\hline 10 & 0.0 & 0.1 & 0.2 & 2.0 & 0.7 \\
\hline 11 & 0.0 & 0.0 & 0.0 & 0.0 & 0.0 \\
\hline
\end{tabular}

a Ordered according to MMFF energy. Only conformers with relative B3LYP/6-31G(d) energies within $6 \mathrm{kcal} / \mathrm{mol}$ are listed. ${ }^{\mathrm{b}}$ Small basis set: B3LYP/6-31G(d); large basis set: B3LYP/6-311G+(d,p) ${ }^{c} \Delta \mathrm{H}$ : populations estimated from internal energies; $\triangle \mathrm{G}$ : populations estimated from ZPC-free energies. ${ }^{\mathrm{d}} \mathrm{PCM}$ model for acetonitrile.

As for the basis set, a double- $\zeta$ Pople's type basis set such as 6$31 \mathrm{G}(\mathrm{d})$ may be unsuited to calculate geometries and relative energies accurately enough, especially due to the intramolecular 
basis set superposition error (BSSE). ${ }^{47-49}$ A larger basis set should be considered at least in single-point or frequency calculations to estimate internal or free energies (step $2 b$ in our flowchart). Time-saving approaches such as the resolution of identity method ${ }^{50}$ or the chain-of-spheres approximation ${ }^{51}$ may be useful in combination with large basis sets. To illustrate how much the calculation method may affect the conformational population even for a relatively rigid organic molecule, we report in Table 1 the Boltzmann population estimated at $300 \mathrm{~K}$ for altersolanol L (2, Scheme 1$)^{46}$ with 5 different methods: (a) MMFF energies from step (1) of the flowchart; (b) internal energies calculated at B3LYP/6-31G(d) level, from step (2a); (c) internal energies calculated at B3LYP/6-311+G(d,p) level; (d) internal energies calculated at the same level as in (c), plus including PCM for acetonitrile, from step (2b); (e) ZPC-free energies calculated at the same level as in (d). The dramatic effect of basis set size and solvent model on the conformer population should warn anyone against the naïve approach of obtaining input structures for ECD (or other properties) calculations just by B3LYP/6-31G(d) geometry optimizations.

Another instructive example of the impact of basis set size on relative energies of a set of conformers, and on an average chiroptical property (in this case optical rotation, OR) calculated thereof, is shown in Figure 3. ${ }^{52}$ The more general take-home message from this example is that when a reasonable change in conformer population affects the sign of average calculated OR (or some diagnostic CD band), the assignment should be doubted, and an alternative method should be considered. ${ }^{53}$

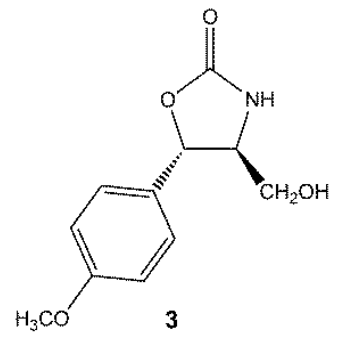

B3LYP/6-31G(d) geometries: 8 confs. ( + ) overall $95 \%$ pop. 2 confs. (-) overall $5 \%$ pop. average $[\alpha]+39$

$B 3 L Y P / 6-31 \mathrm{G}++(d, p)$ geometries: 6 confs. $(4)$ overall $16 \%$ pop. 4 confs. (-) overall $84 \%$ pop. average $[\alpha]-19$

FIGURE 3 Basis-set dependence in the geometry optimization of trans-isocytoxazone $(3)$ and its consequences on calculated OR. ${ }^{52}$

We'd like to mention here a different approach toward AC assignment based on the comparison between the ECD spectrum measured in the solid state, as microcrystalline $\mathrm{KBr}$ or $\mathrm{KCl}$ pellet, and that calculated by TDDFT using as input geometry the X-ray structure (possibly after minimal optimization). This method, known as the solid-state ECD/TDDFT approach, skips steps (1)-(5) in the flowchart and, apart from the time-saving, avoids most of the problems related with a correct geometry prediction; on the other hand, it requires crystals suitable for X-ray analysis. ${ }^{54}$ In the example of Figure 4, the $A C$ of a natural product (schisanterin $A, 4$ ) was established considering the single solid-state geometry instead of the many low-energy conformers (20 distinct DFT minima with relative internal energies $3 \mathrm{kcal} / \mathrm{mol}$ estimated at B3LYP/6-311G+(d,p) level). ${ }^{55}$

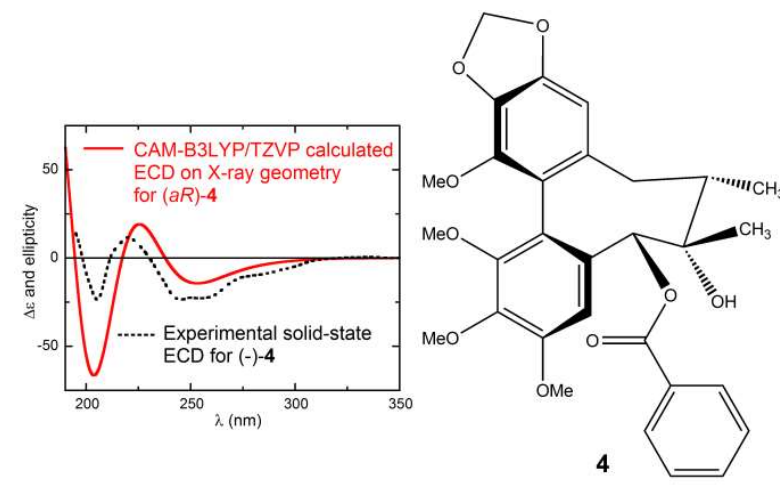

FIGURE 4 AC assignment of schisantherin A (4) by means of the solid-state ECD/TDDFT approach. ${ }^{55}$

\section{Boltzmann Distribution}

The Boltzmann distribution is universally employed to calculate average molecular properties over a set of conformers each of which has its own calculated property, such as a component ECD spectrum..$^{20,30,31}$ One must however be aware that the use of Boltzmann distribution is based on some assumptions: (a) the various conformers are enough separated from each other on the potential energy surface, that is, they lie in sharp energy wells with barriers high enough to keep vibrational states confined to each well; (b) the predicted property (i.e., ECD spectrum) is equivalent for all vibrational states within the energy well. These approximations may easily fail when low-frequency vibrational motions are involved, most notably torsional modes associated with flat potentials, and this may have some consequence on the prediction of ECD and - especially OR. ${ }^{56,57}$

A second point to be considered is the quantity used in the Boltzmann distribution. As said above, geometry optimizations or single-point calculations afford enthalpies or internal energies; subsequent frequency calculations afford ZPC-energies and free energies. In principle these latter should be more reliable than internal energies because they include vibrational entropic factors. An example of the different distribution obtained by using internal and free energies is shown in Table 1 above. In practice, however, calculated entropies may be very inaccurate, especially for low-energy vibrations, and at least a weighting factor should be used to correct the vibrational energies. ${ }^{58,59}$

\section{Choice of the TDDFT Functional}

As we said in the first section, the choice of the functional/basis set combination in TDDFT calculations is the most crucial choice in the whole procedure. On the other hand, this is also one step which can be easily controlled by non-specialists. In fact, the most important thing to do is simply to try different combinations of functionals and basis sets, choosing within a set of popular functionals and reasonable basis sets, and verify the consistency of their results. In fact, the most common and most dangerous error at this step is to use only a single combination, typically again B3LYP with double or triple- $\zeta$ basis set, and take its result for granted. It is important to stress that a more systematic search for optimal calculation level does not need to be run on the whole conformational ensemble, but rather the lowest-energy conformer or a limited set of stable conformations usually suffice, with a considerable time-saving. 
Provided that the "best" functional for the specific problem should be selected, how is this choice made? Unfortunately there is no general answer to this question. When excited state predictions are concerned as with TDDFT calculations, vertical transition energies and absolute oscillator strengths are normally given a primary importance. ${ }^{60-62}$ When full absorption and/or ECD spectra are concerned, however, the relative energy and intensity of the various transition dipole and rotational strengths appear to be more important than absolute values. As a matter of fact, both a wavelength shift (UV correction) and some intensity scaling are normally applied during the computational/ experimental comparison in the last step (9) of the flowchart. Therefore, no universal recipe may be given as for the best performing functional/basis set combination for ECD calculations, and the choice must rely on a trial-and-error approach. Generally speaking, both relative transition wavelengths and intensities depend strongly on the HF fraction of hybrid functionals and on the range separation. Consequently, one should pick up different examples from the two families, as suggested in the previous section, and test each of them on one or more selected structures, in association with a good basis set, for example def2-SVP or def2-TZVP. The functional(s) performing better in terms of overall spectrum appearance should be then selected for further basis set testing (if necessary), and finally to run the calculations on the remaining conformers.

In the following we will discuss a few examples which aim at demonstrating the impact of the functional on the quality of the final result, stressing especially the comparison between B3LYP and other functionals.
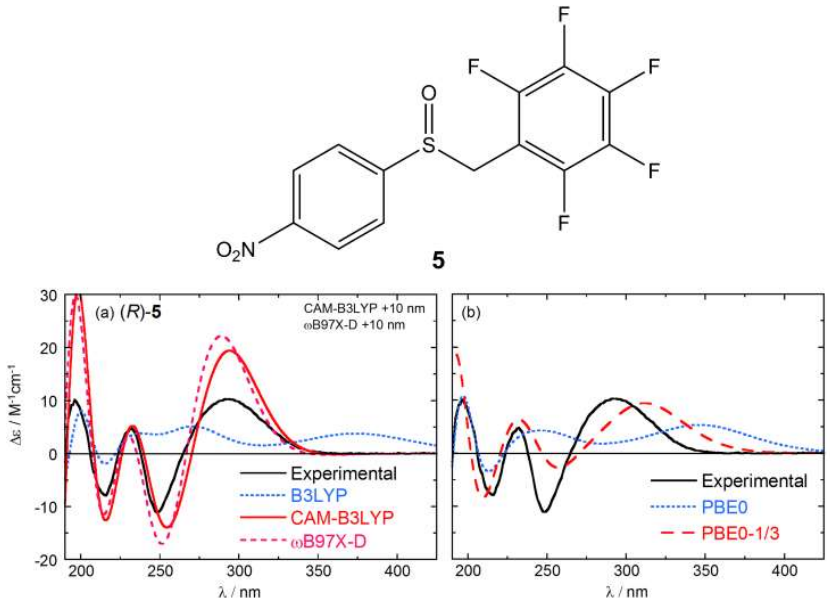

FIGURE 5 Comparison of experimental and TDDFT-calculated ECD spectra for sulfoxide $\mathbf{5}$ with different functionals. All TDDFT calculations were run with the same basis set (TZVP) using PCM model for acetonitrile. The calculated spectra are the Boltzmann average over 3 structures, geometry optimized at B3LYP/6$311+G(d, p)$ level in acetonitrile, and UV-corrected. ${ }^{63}$

Several recent reports demonstrate that range-separated functionals like CAM-B3LYP and $\omega B$ 97X-D are superior to hybrid functionals, first of all B3LYP, in the prediction of chiroptical properties ${ }^{63-65} \mathrm{~A}$ good example is offered by sulfoxide 5 (Figure 4), for which CAM-B3LYP and $\omega B$ 97X-D were able to predict the correct sequence of ECD bands, while B3LYP was not, particularly because of a severe underestimation of the energy of the first band. ${ }^{63}$ When such a situation occurs, a deeper analysis of transitions and orbitals is desirable to uncover a possible specific functional-related issue. In the case of compound $\mathbf{5}$ and its analogs, as well as of the bisporphyrin system $\mathbf{6}$ discussed below, the major responsible for the failure of B3LYP is the large charge-transfer (CT) character of the "missed" transition(s). B3LYP and many other hybrid functionals are known to perform poorly for CT-like transitions because of an incorrect asymptotic behavior. ${ }^{62,66,67}$ This problem may be evidenced by evaluating the CT character of specific transitions by a metric measurement, ${ }^{36,68}$ and conveniently tackled e.g. by using a range-separated functional. ${ }^{36,67}$

The same example is also useful to point out the dependence of TDDFT ECD calculations on the exact exchange fraction in hybrid DFT functionals. In fact, a small increase in the amount of $\mathrm{HF} \%$, i.e. passing from $25 \%$ of PBE0 to $33 \%$ of PBE0-1/3, 69 significantly improved the performance of the PBE-based hybrid functional (Figure 5b). This demonstrates how useful it can be to compare similar functionals with different $\mathrm{HF} \%$, for example B3LYP $(20 \% \mathrm{HF})$ vs. BH\&HLYP (50\%, also known as BHLYP), PBE0 (25\%) vs. PBE0-1/3 (33\%), M06 (27\%) vs. M06-2X (54\%) and so on.

Another illustrative case is that of the bis(aminoporphyrin) 6, for which both the geometry optimization and the TDDFT calculations were sensitive to the used functional. ${ }^{70}$ The phenyl rings adjacent to the interporphyrin axis lie above the neighboring porphyrinic macrocycle. The resulting $\pi$-stacking is correctly reproduced only by dispersion-corrected functionals such as $\omega B 97 X-D$. The $\pi$-stacking has a strong influence on the dihedral angle of the axis and thus on the ECD spectrum of the found conformers. ${ }^{70}$ Moreover, CAM-B3LYP, B3LYP, and $B H L Y P$ predict quite different ECD spectra with only the longrange corrected functional CAM-B3LYP reproducing the correct pattern, intensities, and signs of the experimental curve (Figure 5). B3LYP in particular performed poorly due to the already discussed CT-related issue.

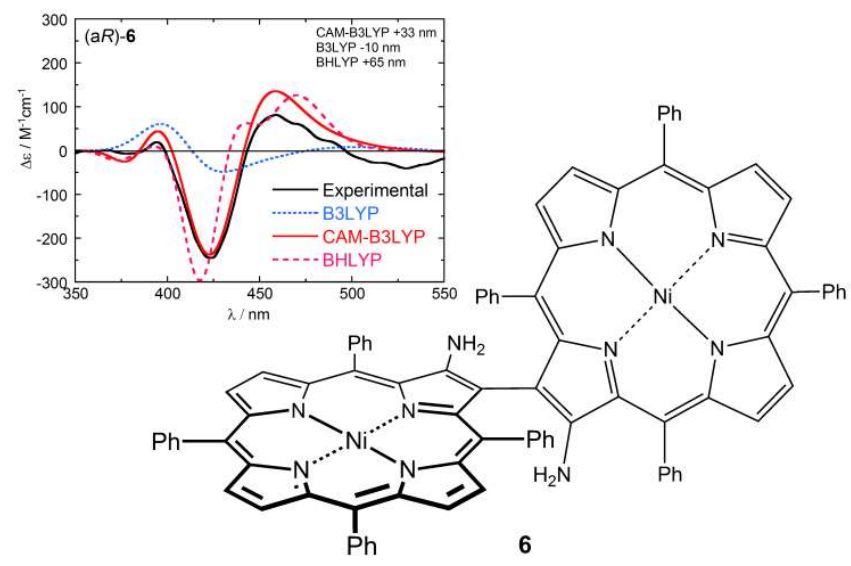

FIGURE 6 Comparison of experimental (intensity scaled to that of the CAM-B3LYP curve) and TDDFT-calculated ECD spectra of the main conformer of the aminoporphyrin dimer 6 calculated with B3LYP, CAM-B3LYP, and BHLYP. All computations were run with the same basis set $\left(6-31 \mathrm{G}^{*}\right.$ for $\mathrm{C}, \mathrm{H}$, and $\mathrm{N}$ and $6-311 \mathrm{G}^{*}$ for $\left.\mathrm{Ni}\right)$ and with $\mathrm{CPCM}$ for dichloromethane. Optimization was done with $\omega B 97 X-D / 6-31 G^{*}\left(6-311 G^{*}\right.$ for $\left.\mathrm{Ni}\right)$ and the spectra are UV-corrected.

\section{Choice of the TDDFT Basis Set}

The choice of the basis set for the TDDFT calculations is in principle less critical than for the calculation of free energies. The oscillator and rotational strength values are not very sensitive to basis set, moreover two simple pragmatic approaches may help addressing this point. First, rotational strengths calculated from dipole length (DL) and velocity (DV) formalisms converge to each other when approaching basis set completeness, ${ }^{71}$ and 
most software packages provide results for DL and DV rotational strengths for a direct comparison. Second, one may run several TDDFT calculations on one representative conformer keeping the functional fixed and increasing the basis set size within one family, e.g. using SVP, TZVP, minimally augmented TZVP (maTZVP) and augmented TZVP (aug-TZVP), ${ }^{26,72}$ and look for the best cost-accuracy compromise. Often double or triple- $\zeta$ nonaugmented basis sets already give reliable results, but diffuse functions may be required when Rydberg states are involved, ${ }^{37}$ or, in general, for anionic species. Apart from the computational cost, diffuse functions often lead to severe problems with linear dependencies in the SCF, thus posing convergence issues. A good compromise are the so-called minimally augmented basis sets (e.g. ma-TZVP, ma-cc-pVTZ) suggested by Truhlar et al., ${ }^{72}$ which significantly lower computational costs at nearly the same accuracy as the large augmented basis sets. ${ }^{73}$

A more difficult and thus more decisive point is the computation of the excitation energies, as they show a higher sensitivity towards the basis set, depending on the nature of the chromophore(s) under investigation. To get the correct absolute values the basis set needs to be very large, including polarization and diffuse functions, keeping in mind that transition energies also depend strongly on the functional. ${ }^{60-62}$ However, as said above, for the determination of the absolute configuration it is not necessary to get the correct absolute energy values, instead the relative energies must be reliable. The systematic shift between experimental and weighted-average spectra is usually larger for smaller basis sets, but the UV correction mentioned in step (9) is sufficient to handle it.

\section{Solvent model}

The impact of solvent on ECD spectroscopy is usually less dramatic than for other chiroptical spectroscopies such as OR, VCD and ROA. Still, solvent may affect both the relative stability and the component ECD spectrum for each conformer. ${ }^{74}$ In the course of both DFT geometry optimizations and TDDFT calculations, non-specific solute-solvent interactions may be accounted for with a continuum solvent model such as $\mathrm{PCM}^{27}$ or COSMO,$^{28}$ whose computational cost is often very reasonable. On the contrary, specific solute-solvent, solute-solute or intramolecular interactions require the use of explicit solvation models, ${ }^{74}$ which are more demanding.

It is impossible to discuss solvent effects in any exhaustive way in the present contribution. Even the apparently simple question of whether one should consider or not a solvent model in ECD calculations is far from trivial. Staying on a very pragmatic ground, we recommend the use of a continuum solvent model in single-point calculations of conformational energies (step (3) in the flowchart), and possibly in TDDFT calculations for some selected conformers, at least when moderately polar molecules are concerned in combination with polar solvents. An example of impact of PCM on a conformational population is given in Table 1. As for TDDFT calculations, PCM affects especially relative transition energies and may provide a better spacing between calculated ECD bands.

The inclusion of an explicit solvent model should be considered only when other things fail. For example, in step (5) of the flowchart, the calculated conformational ensemble may contradict independent experimental findings, e.g. NMR data. Very often this is due to missing intramolecular interactions like $\pi$-stacking or hydrogen bonds occurring in solution, or strong specific solute-solvent interactions such as hydrogen bonding in water. Their correct simulation needs explicit solvent molecules to be considered in the conformational search, in the geometry optimization and in the TDDFT calculation steps. For examples of such a procedure, which may easily get very complicated and computationally expensive, we refer the interested reader to the literature. ${ }^{74-77}$

\section{Beyond TDDFT}

We have only discussed TDDFT as the method of choice for running ECD calculations, in view of its present great popularity. However, several other methods are feasible for excited-state calculations which represent useful alternatives to TDDFT especially in some circumstances. ${ }^{10,11,13,33,34}$ Although conventional TDDFT is very time-effective, it is not feasible to calculate the ECD of larger molecules. Here the newly developed simplified TDDFT (sTDDFT) method is a very efficient alternative and allows ECD calculations of molecules with up to 1000 atoms at limited computational cost with good accuracy. ${ }^{78}$ Another problem for TDDFT are chromophores with severe longrange $\mathrm{CT}$ excitations which can hardly be handled by any functional. Other methods like SCS-CIS(D) (spin-componentscaled configuration interaction singles with perturbative doubles correction), or the coupled-cluster approaches CC2 or EOMCCSD (equation-of-motion coupled-cluster singles and doubles) may be employed in these cases. ${ }^{79,80} \mathrm{CC} 2$ in particular has received much attention in ECD calculations. ${ }^{81-83}$ Like TDDFT, coupled-cluster methods cannot handle multi-reference wave functions. The use of $\mathrm{MRCl}$ (multi-reference configuration interaction) techniques becomes necessary for molecules not fully described by a single electronic configuration. ${ }^{84}$ However, these methods are very time demanding and especially $\mathrm{MRCl}$ should be applied only by experienced users.

\section{Conclusion}

Quantum-mechanical calculations of chiroptical properties are nowadays an easily accessible and powerful tool for assigning absolute configurations of several classes of molecules, in particular small or medium-sized natural products. However, good practice in TDDFT calculations of ECD spectra needs to be more widespread, both to avoid unwanted errors in $A C$ assignments and to favour a more critical use of computational methods. From our discussion it should be clear enough that no computational recipe is universally valid in all cases and for all molecules. In particular, the very popular "B3LYP/6-31G(d)" approach should be discouraged in general, although it may well provide the correct AC assignment. The most important message from the present contribution is that every method of calculation is prone to error, therefore one should never limit to a single level of calculation and should always explore different combinations of e.g. DFT functionals and basis sets. A second crucial point is, in our opinion, that an entirely computational approach is insufficient, especially in the generation of input structures. The conformational ensemble obtained in silico should always be checked against all available experimental data from other techniques such as NMR. We also recommend an independent assessment of relative configuration by means of X-ray diffractometry or again NMR, through a complete set of $\mathrm{J}$-couplings and NOE measurements, whose interpretation is supported by molecular modeling. Finally, in the final comparison between the experimental and the computed ECD spectrum, it is 
indispensable not to focus on a single band or a narrow wavelength window, but rather to consider the whole available spectral range. In the same step, a quantitative spectral comparison is also to be preferred over a merely visual one.

\section{Acknowledgements}

We are grateful to all our co-workers and co-authors in the field. Dr. Marcin Górecki is thanked for useful discussions.

\section{REFERENCES}

1. Mack CA. Fifty Years of Moore's Law. IEEE Trans Semiconduct Manufact 2011;24:202-207.

2. Eliel EL, Wilen SH. Stereochemistry of organic compounds. New York: Wiley; 1994.

3. Comprehensive Chiroptical Spectroscopy. Berova N, Polavarapu PL, Nakanishi K, Woody RW, editors. Hoboken (NJ): John Wiley \& Sons, Inc.; 2012.

4. Circular Dichroism. Principles and Applications. Nakanishi K, Berova N, Woody RW, editors. New York: VCH; 1994.

5. Fundamentals Aspects and Recent Developments in Optical Rotatory Dispersion and Circular Dichroism. Ciardelli F, Salavdori P, editors. London: Heiden; 1973.

6. Harada N, Nakanishi K. Circular Dichroic Spectroscopy - Exciton Coupling in Organic Stereochemistry. Mill Valley, CA: University Science Books; 1983.

7. Harada N, Nakanishi K, Berova N. Electronic CD Exciton Chirality Method: Principles and Applications. In: Berova N, Polavarapu PL, Nakanishi K, Woody RW, editors. Comprehensive Chiroptical Spectroscopy. Hoboken (NJ): John Wiley \& Sons, Inc.; 2012. p 115-166.

8. Polavarapu PL. Renaissance in chiroptical spectroscopic methods for molecular structure determination. Chem Rec 2007;7:125-136.

9. Autschbach J. Computing chiroptical properties with first-principles theoretical methods: Background and illustrative examples. Chirality 2009;21:E116-E152.

10. Goerigk L, Kruse H, Grimme S. Theoretical Electronic Circular Dichroism Spectroscopy of Large Organic and Supramolecular Systems. In: Berova N, Polavarapu PL, Nakanishi K, Woody RW, editors. Comprehensive Chiroptical Spectroscopy. Hoboken (NJ): John Wiley \& Sons, Inc.; 2012. p 643-673.

11. Autschbach J. Ab Initio Electronic circular dichroism and optical rotatory dispersion: from organic molecules to transition metal complexes. In: Berova N, Polavarapu PL, Nakanishi K, Woody RW, editors. Comprehensive Chiroptical Spectroscopy. Hoboken (NJ): John Wiley \& Sons, Inc.; 2012. p 593-642.

12. Autschbach J, Nitsch-Velasquez L, Rudolph M. Time-Dependent Density Functional Response Theory for Electronic Chiroptical Properties of Chiral Molecules. In: Naaman R, Beratan ND, Waldeck D, editors. Electronic and Magnetic Properties of Chiral Molecules and Supramolecular Architectures. Berlin, Heidelberg: Springer Berlin Heidelberg; 2011. p 1-98.

13. Warnke I, Furche F. Circular dichroism: electronic. WIREs Comput Mol Sci 2012;2:150-166.

14. Autschbach J, Nitsch-Velasquez L, Rudolph M. Time-Dependent Density Functional Response Theory for Electronic Chiroptical Properties of Chiral Molecules. Top Curr Chem 2011;298:1-98.
15. Nugroho AE, Morita $H$. Circular dichroism calculation for natural products. J Nat Med 2013;68:1-10.

16. Polavarapu PL. Molecular Structure Determination Using Chiroptical Spectroscopy: Where We May Go Wrong? Chirality 2012;24:909-920.

17. Junior FMS, Covington CL, de Amorim MB, Velozo LSM, Kaplan MAC, Polavarapu PL. Absolute Configuration of a Rare Sesquiterpene: (+)-3-Ishwarone. J Nat Prod 2014;77:1881-1886.

18. Derewacz DK, McNees CR, Scalmani G, Covington CL, Shanmugam G, Marnett LJ, Polavarapu PL, Bachmann BO. Structure and Stereochemical Determination of Hypogeamicins from a Cave-Derived Actinomycete. J Nat Prod 2014;77:17591763.

19. Bifulco G, Dambruoso P, Gomez-Paloma L, Riccio R. Determination of Relative Configuration in Organic Compounds by NMR Spectroscopy and Computational Methods. Chem Rev 2007;107:3744-3779.

20. Bringmann G, Bruhn T, Maksimenka K, Hemberger $Y$. The Assignment of Absolute Stereostructures through Quantum Chemical Circular Dichroism Calculations. Eur J Org Chem 2009:2717-2727.

21. Halgren TA. Merck molecular force field. I. Basis, form, scope, parameterization, and performance of MMFF94. J Comput Chem 1996;17:490-519 and following papers.

22. Hinchliffe A. Molecular Modelling for Beginners. Chichester: Wiley; 2008.

23. Brémond É, Savarese $M, S u$ NQ, Pérez-Jiménez ÁJ, Xu X, Sancho-García JC, Adamo C. Benchmarking Density Functionals on Structural Parameters of Small-/Medium-Sized Organic Molecules. J Chem Theory Comput 2016:Article ASAP, DOI: 10.1021/acs.jctc.5b01144.

24. Grimme S, Steinmetz M. Effects of London dispersion correction in density functional theory on the structures of organic molecules in the gas phase. Phys Chem Chem Phys 2013;15:16031-16042.

25. Grimme S, Brandenburg JG, Bannwarth C, Hansen A. Consistent structures and interactions by density functional theory with small atomic orbital basis sets. J Chem Phys 2015;143:054107.

26. Weigend F, Ahlrichs R. Balanced basis sets of split valence, triple zeta valence and quadruple zeta valence quality for $\mathrm{H}$ to $\mathrm{Rn}$ : Design and assessment of accuracy. Phys Chem Chem Phys 2005; 7:3297-3305.

27. Tomasi J, Mennucci B, Cammi R, editors. Continuum Solvation Models in Chemical Physics: From Theory to Applications Chichester: Wiley; 2007.

28. Klamt A, Schuurmann G. COSMO: a new approach to dielectric screening in solvents with explicit expressions for the screening energy and its gradient. J Chem Soc Perkin Trans 2 1993:799-805.

29. Marenich AV, Cramer CJ, Truhlar DG. Universal Solvation Model Based on Solute Electron Density and on a Continuum Model of the Solvent Defined by the Bulk Dielectric Constant and Atomic Surface Tensions. J Phys Chem B 2009;113:6378-6396.

30. Freedman TB, Cao X, Dukor RK, Nafie LA. Absolute configuration determination of chiral molecules in the solution state using vibrational circular dichroism. Chirality 2003;15:743-758.

31. Pescitelli G, Di Bari L, Berova N. Conformational Aspects in the Studies of Organic Compounds by Electronic Circular Dichroism. Chem Soc Rev 2011;40:4603-4625. 
32. Mazzanti A, Casarini D. Recent trends in conformational analysis. WIREs Comput Mol Sci 2012;2:613-641.

33. Diedrich C, Grimme S. Systematic Investigation of Modern Quantum Chemical Methods to Predict Electronic Circular Dichroism Spectra. J Phys Chem A 2003;107:2524-2539.

34. Crawford TD, Tam MC, Abrams ML. The Current State of Ab Initio Calculations of Optical Rotation and Electronic Circular Dichroism Spectra. J Phys Chem A 2007;111:12057-12068.

35. All references about DFT functionals and basis sets can be found in the on-line documentation for Gaussian'09 at www.gaussian.com/g_tech/g_ur/k_dft.htm and www.gaussian.com/g_tech/g_ur/m_basis_sets.htm respectively. However, functional definitions may differ from software to software.

36. Le Bahers T, Adamo C, Ciofini I. A Qualitative Index of Spatial Extent in Charge-Transfer Excitations. J Chem Theory Comput 2011; 7:2498-2506.

37. Reinscheid F, Reinscheid UM. Stereochemical analysis of (+)limonene using theoretical and experimental NMR and chiroptical data. J Mol Struct 2016;1106:141-153.

38. Stephens PJ, Harada N. ECD cotton effect approximated by the Gaussian curve and other methods. Chirality 2010;22:229-233.

39. Bruhn T, Schaumlöffel A, Hemberger Y, Bringmann G. SpecDis: Quantifying the Comparison of Calculated and Experimental Electronic Circular Dichroism Spectra. Chirality 2013;25:243-249.

40. Polavarapu PL, Covington CL. Comparison of Experimental and Calculated Chiroptical Spectra for Chiral Molecular Structure Determination. Chirality 2014;26:539-552.

41. Sousa SF, Fernandes PA, Ramos MJ. General Performance of Density Functionals. J Phys Chem A 2007;111:10439-10452.

42. Zhao Y, Truhlar DG. Density Functionals with Broad Applicability in Chemistry. Acc Chem Res 2008;41:157-167.

43. Chai J-D, Head-Gordon M. Systematic optimization of long-range corrected hybrid density functionals. J Chem Phys 2008;128:084106.

44. Kruse H, Goerigk L, Grimme S. Why the Standard B3LYP/6$31 G^{*}$ Model Chemistry Should Not Be Used in DFT Calculations of Molecular Thermochemistry: Understanding and Correcting the Problem. J Org Chem 2012;77:10824-10834.

45. Goerigk L, Grimme S. Double-hybrid density functionals. WIREs Comput Mol Sci 2014;4:576-600.

46. Debbab A, Aly AH, Edrada-Ebel R, Wray V, Pretsch A, Pescitelli G, Kurtan T, Proksch P. New Anthracene Derivatives - Structure Elucidation and Antimicrobial Activity. Eur J Org Chem 2012:13511359.

47. Riley KE, Op't Holt BT, Merz KM. Critical Assessment of the Performance of Density Functional Methods for Several Atomic and Molecular Properties. J Chem Theory Comput 2007;3:407433.

48. Balabin RM. Enthalpy difference between conformations of normal alkanes: Intramolecular basis set superposition error (BSSE) in the case of $n$-butane and $n$-hexane. J Chem Phys 2008; 129:164101.

49. Sure R, Brandenburg JG, Grimme S. Small Atomic Orbital Basis Set First-Principles Quantum Chemical Methods for Large Molecular and Periodic Systems: A Critical Analysis of Error Sources. ChemistryOpen 2015:Early View, DOI: 10.1002/open.201500192.
50. Xinguo R, Patrick R, Volker B, Jürgen W, Alexandre T, Andrea S, Karsten R, Matthias S. Resolution-of-identity approach to HartreeFock, hybrid density functionals, RPA, MP2 and GW with numeric atom-centered orbital basis functions. New J Phys 2012;14:053020.

51. Neese F, Wennmohs F, Hansen A, Becker U. Efficient, approximate and parallel Hartree-Fock and hybrid DFT calculations. A 'chain-of-spheres' algorithm for the Hartree-Fock exchange. Chem Phys 2009;356:98-109.

52. Mazzeo G, Giorgio E, Zanasi R, Berova N, Rosini C. Absolute Configuration through the DFT Simulation of the Optical Rotation. Importance of the Correct Selection of the Input Geometry: A Caveat. J Org Chem 2010;75:4600-4603.

53. Polavarapu PL. Why is it important to simultaneously use more than one chiroptical spectroscopic method for determining the structures of chiral molecules? Chirality 2008;20:664-672.

54. Pescitelli G, Kurtán T, Flörke U, Krohn K. Absolute Structural Elucidation of Natural Products-A Focus on Quantum-Mechanical Calculations of Solid-State CD Spectra. Chirality 2009;21:E181E201.

55. Liu H-W, Yu X-Z, Padula D, Pescitelli G, Lin Z-W, Wang F, Ding $\mathrm{K}$, Lei M, Gao J-M. Lignans from Schisandra sphenathera Rehd. et Wils. and semisynthetic schisantherin A analogues: Absolute configuration, and their estrogenic and anti-proliferative activity. Eur J Med Chem 2013;59:265-273.

56. Crawford TD, Allen WD. Optical activity in conformationally flexible molecules: a theoretical study of large-amplitude vibrational averaging in $(<\mathrm{i}>\mathrm{R}<\mathrm{i}>\mathrm{)})-3$-chloro-1-butene. Mol Phys 2009;107:1041 - 1057 .

57. Mort BC, Autschbach J. Magnitude of Zero-Point Vibrational Corrections to Optical Rotation in Rigid Organic Molecules: A Time-Dependent Density Functional Study. J Phys Chem A 2005;109:8617-8623.

58. Laury ML, Boesch SE, Haken I, Sinha P, Wheeler RA, Wilson AK. Harmonic vibrational frequencies: Scale factors for pure, hybrid, hybrid meta, and double-hybrid functionals in conjunction with correlation consistent basis sets. J Comput Chem 2011;32:23392347.

59. Grimme S, Mück-Lichtenfeld C. Calculation of conformational energies and optical rotation of the most simple chiral alkane. Chirality 2008;20:1009-1015.

60. Jacquemin D, Perpète EA, Scuseria GE, Ciofini I, Adamo C. TDDFT Performance for the Visible Absorption Spectra of Organic Dyes: Conventional versus Long-Range Hybrids. J Chem Theory Comput 2008;4:123-135.

61. Laurent $A D$, Jacquemin D. TD-DFT benchmarks: A review. Int J Quantum Chem 2013;113:2019-2039.

62. Adamo C, Jacquemin D. The calculations of excited-state properties with Time-Dependent Density Functional Theory. Chem Soc Rev 2013;42:845-856.

63. Berardozzi R, Guido CA, Capozzi MAM, Cardellicchio C, Di Bari L, Pescitelli G. Circular Dichroism and TDDFT Investigation of Chiral Fluorinated Aryl Benzyl Sulfoxides. Eur J Org Chem 2015:5554-5562.

64. Rudolph M, Autschbach J. Performance of Conventional and Range-Separated Hybrid Density Functionals in Calculations of Electronic Circular Dichroism Spectra of Transition Metal Complexes. J Phys Chem A 2011;115:14677-14686. 
65. Jorge FE, Jorge SS, Suave RN. Electronic Circular Dichroism of Chiral Alkenes: B3LYP and CAM-B3LYP Calculations. Chirality 2015;27:23-31.

66. Dreuw A, Head-Gordon M. Failure of Time-Dependent Density Functional Theory for Long-Range Charge-Transfer Excited States: The Zincbacteriochlorin-Bacteriochlorin and Bacteriochlorophyll-Spheroidene Complexes. J Am Chem Soc 2004; 126:4007-4016.

67. Dreuw A, Head-Gordon M. Single-Reference ab Initio Methods for the Calculation of Excited States of Large Molecules. Chem Rev 2005;105:4009-4037.

68. Guido CA, Cortona P, Adamo C. Effective electron displacements: A tool for time-dependent density functional theory computational spectroscopy. J Chem Phys 2014;140:104101.

69. Guido CA, Brémond E, Adamo C, Cortona P. Communication: One third: A new recipe for the PBE0 paradigm. J Chem Phys 2013;138:021104.

70. Bruhn T, Witterauf F, Götz DCG, Grimmer CT, Würtemberger M, Radius U, Bringmann G. C,C- and N,C-Coupled Dimers of 2Aminotetraphenylporphyrins: Regiocontrolled Synthesis, Spectroscopic Properties, and Quantum-Chemical Calculations. Chem Eur J 2014;20:3998-4006.

71. Pecul M, Ruud K, Helgaker T. Density functional theory calculation of electronic circular dichroism using London orbitals. Chem Phys Lett 2004;388:110-119.

72. Zheng J, Xu X, Truhlar DG. Minimally augmented Karlsruhe basis sets. Theor Chem Acc 2010;128:295-305.

73. Papajak E, Truhlar DG. Efficient Diffuse Basis Sets for Density Functional Theory. J Chem Theory Comput 2010;6:597-601.

74. Mennucci B, Cappelli C, Cammi R, Tomasi J. Modeling solvent effects on chiroptical properties. Chirality 2011;23:717-729.

75. Iwahana S, lida H, Yashima E, Pescitelli G, Di Bari L, Petrovic AG, Berova N. Absolute Stereochemistry of a 4 aHydroxyriboflavin Analogue of the Key Intermediate of the FADMonooxygenase Cycle. Chem Eur J 2014;20:4386-4395.

76. Pikulska A, Hopmann KH, Bloino J, Pecul M. Circular Dichroism and Optical Rotation of Lactamide and 2-Aminopropanol in Aqueous Solution. J Phys Chem B 2013;117:5136-5147.

77. Fleming AM, Orendt AM, He Y, Zhu J, Dukor RK, Burrows CJ. Reconciliation of Chemical, Enzymatic, Spectroscopic and Computational Data To Assign the Absolute Configuration of the DNA Base Lesion Spiroiminodihydantoin. J Am Chem Soc 2013;135:18191-18204.

78. Bannwarth C, Grimme S. A simplified time-dependent density functional theory approach for electronic ultraviolet and circular dichroism spectra of very large molecules. Comput Theor Chem 2014;1040-1041:45-53.

79. Goerigk L, Grimme S. Quantum Chemical Investigation of Exciton Coupling: Super-Molecular Calculations of a Merocyanine Dimer Aggregate. ChemPhysChem 2008;9:2467-2470.

80. Sneskov K, Christiansen O. Excited state coupled cluster methods. WIREs Comput Mol Sci 2012;2:566-584.

81. Nakai Y, Nishizaka M, Yang C, Fukuhara G, Mori T, Inoue $Y$. Experimental and theoretical investigations of circular dichroism of donor-acceptor 1,1' -binaphthyls: Influence of substitution on the coupling amplitude and cotton effect of the charge-transfer band. Chirality 2011;23:E22-E27.
82. Nakai $\mathrm{Y}$, Mori T, Sato K, Inoue $\mathrm{Y}$. Theoretical and Experimental Studies of Circular Dichroism of Mono- and Diazonia[6]helicenes. J Phys Chem A 2013;117:5082-5092.

83. Bruhn T, Pescitelli G, Jurinovich $S$, Schaumlöffel A, Witterauf $F$, Ahrens J, Bröring M, Bringmann G. Axially Chiral BODIPY DYEmers: An Apparent Exception to the Exciton Chirality Rule. Angew Chem Int Ed 2014;53:14592-14595.

84. Brückner C, Götz DCG, Fox SP, Ryppa C, McCarthy JR, Bruhn T, Akhigbe J, Banerjee S, Daddario P, Daniell HW, Zeller M, Boyle RW, Bringmann G. Helimeric Porphyrinoids: Stereostructure and Chiral Resolution of meso-Tetraarylmorpholinochlorins. J Am Chem Soc 2011;133:8740-8752. 